\title{
A!
}

This is an electronic reprint of the original article.

This reprint may differ from the original in pagination and typographic detail.

Zeng, Hua Bi; Tian, Yu; Fan, Zheyong; Chen, Chiang Mei

\section{Nonlinear transport in a two dimensional holographic superconductor}

Published in:

Physical Review D

DOI:

10.1103/PhysRevD.93.121901

Published: 16/06/2016

Document Version

Publisher's PDF, also known as Version of record

Please cite the original version:

Zeng, H. B., Tian, Y., Fan, Z., \& Chen, C. M. (2016). Nonlinear transport in a two dimensional holographic superconductor. Physical Review D, 93(12), 1-5. [121901]. https://doi.org/10.1103/PhysRevD.93.121901

This material is protected by copyright and other intellectual property rights, and duplication or sale of all or part of any of the repository collections is not permitted, except that material may be duplicated by you for your research use or educational purposes in electronic or print form. You must obtain permission for any other use. Electronic or print copies may not be offered, whether for sale or otherwise to anyone who is not an authorised user. 


\title{
Nonlinear transport in a two dimensional holographic superconductor
}

\author{
Hua Bi Zeng, ${ }^{1,2,3, *}$ Yu Tian, ${ }^{4,5, \dagger}$ Zhe Yong Fan, ${ }^{6, \ddagger}$ and Chiang-Mei Chen ${ }^{2, \S}$ \\ ${ }^{1}$ College of Physics Science and Technology, Yangzhou University, Jiangsu 225009, China \\ ${ }^{2}$ Department of Physics, National Central University, Chungli 32001, Taiwan \\ ${ }^{3}$ School of Mathematics and Physics, Bohai University, JinZhou 121000, China \\ ${ }^{4}$ School of Physics, University of Chinese Academy of Sciences, Beijing 100049, China \\ ${ }^{5}$ Shanghai Key Laboratory of High Temperature Superconductors, Shanghai 200444, China \\ ${ }^{6}$ COMP Centre of Excellence, Department of Applied Physics, Aalto University, P.O. Box 11100, FI-00076 \\ Helsinki, Finland
}

(Received 6 May 2016; published 16 June 2016)

\begin{abstract}
The problem of nonlinear transport in a two-dimensional superconductor with an applied oscillating electric field is solved by the holographic method. The complex conductivity can be computed from the dynamics of the current for both the near- and nonequilibrium regimes. The limit of weak electric field corresponds to the near-equilibrium superconducting regime, where the charge response is linear and the conductivity develops a gap determined by the condensate. A larger electric field drives the system into a superconducting nonequilibrium steady state, where the nonlinear conductivity is quadratic with respect to the electric field. Increasing the amplitude of the applied electric field results in a far-from-equilibrium nonsuperconducting steady state with a universal linear conductivity of one. In the lower temperature regime we also find chaotic behavior of the superconducting gap, which results in a nonmonotonic fielddependent nonlinear conductivity.
\end{abstract}

DOI: 10.1103/PhysRevD.93.121901

\section{INTRODUCTION}

Charge transport of a system under a perturbative electric field $E \cos (\omega t)$ can be well understood by the linear response/Kubo formalism, since the properties of the system will be hardly affected by a small $E$. Nonlinear transport occurs naturally if we keep increasing the strength of the applied electric field, and the properties of the system depend in a singular way on $E$. However, understanding the nonlinear transport calls for a theory beyond the linear response theory, which is always a difficult task [1,2], except for a system close to a quantum critical point where the appropriate nonequilibrium Green function and induced current can be calculated [3-8]. Therefore, new insights and a general method are needed for studying a system with an arbitrary strength of field and away from a critical point.

The AdS/CFT correspondence [9-12] states that the dynamics of a quantum many-body system can be obtained by solving the classical time evolution equation of its gravity dual, no matter whether the system is near or even far from equilibrium. For example, holography has been applied successfully to get insights about the superconducting gap dynamics for a long time evolution and far from equilibrium state in both a spatial homogeneous configuration [13-19] and an inhomogeneous configuration [20-23]. Many efforts have also been devoted to studying the superconducting equilibrium state phase transitions and

\footnotetext{
*zenghbi@gmail.com

†tian@ucas.ac.cn

brucenju@gmail.com

§cmchen@phy.ncu.edu.tw
}

charge transport properties in the linear response regime by following Refs. [24,25]; for a review, see Ref. [26]. The applications of holography to condensed matter are now known as the AdS/CMT correspondence [27-30].

In condensed matter literature, previous works on the nonlinear charge transport in two-dimensional superconductors mainly focused on the situation close to zero-temperature quantum critical points between the superconducting and insulating states when a constant electric field is applied [1,3]. The holographic study of nonlinear conductivity focused on a nonsuperconducting steady current driven by a constant or oscillating electric field [5,6,31] and an $E$ - and $\omega$-independent constant nonlinear conductivity was found. However, an investigation of the nonlinear complex conductivity corresponding to an oscillating electric field away from the equilibrium state is still lacking. An electric field like $E \cos (\omega t)$ will induce a time-dependent pair-breaking current in the superconductor. The linear response theory can only address the regime of a very small $E$ in a static superconducting background. A large $E$ can induce a larger current which will drive the system out of equilibrium and suppress the superconducting gap, and eventually destroy superconductivity via a nonequilibrium phase transition [17].

Since holography provides an applicable method to easily compute the current induced by an external field, we extend the framework of the holographic superconductor to study the real-time dynamics of current in both nearequilibrium and far-from-equilibrium regimes. With this method we are able to study nonlinear charge transport in the nonequilibrium regime, which is beyond the capability 
ZENG, TIAN, FAN, and CHEN

of the linear response theory. A specific $E$-dependent conductivity, $\sigma(\omega, E) \sim E^{2}$, appears in the far-infrared regime where the electric field can suppress the superconducting gap but is not strong enough to destroy it. By increasing $E$, the superconductivity will be destroyed at a critical value, and then the conductivity approaches a universal value of one.

\section{MODEL: CURRENT DYNAMICS AND NONEQUILIBRIUM PHASE TRANSITION}

The action of a $s$-wave holographic superconductor includes a $U(1)$ gauge field and a charged scalar,

$$
S=\int d^{4} x \sqrt{-g}\left(-\frac{1}{4} F_{\mu \nu} F^{\mu \nu}-|\nabla \Psi-i A \Psi|^{2}-m^{2}|\Psi|^{2}\right),
$$

where we choose the mass parameter $m^{2}=-2$ without loss of generality. The background is assumed to be the neutral $\mathrm{AdS}_{4}$ planar black hole and its metric, in the retarded Eddington coordinates, reads

$$
d s^{2}=\frac{1}{z^{2}}\left(-f(z) d t^{2}-2 d t d z+d x^{2}+d y^{2}\right),
$$

where $f(z)=1-z^{3}$. The location of the horizon is at $z=1$, while $z=0$ is the boundary where the field theory lives. According to the holographic dictionary, the gauge field in the bulk will source a conserved current $J$ on the boundary, while the scalar will source a scalar operator $O$ which breaks the $U(1)$ symmetry of the boundary field theory. Specifically, the asymptotical behaviors of the bulk fields on the boundary are

$$
\Psi=\Psi^{(1)} z+\Psi^{(2)} z^{2}, \quad A_{\mu}=a_{\mu}+b_{\mu} z .
$$

In the alternative quantization, the source term $\Psi^{(1)}$ is switched off to guarantee the appearance of a spontaneous symmetry-broken phase by a nonvanishing $\Psi^{(2)}$, and the expectation values of $O$ and $J$ are obtained by holography as the variation of the renormalized bulk on-shell action with respect to the sources [17], i.e.,

$$
\langle O\rangle=\Psi^{(2)}, \quad J_{\mu}=-b_{\mu}+\partial_{t} a_{\mu} .
$$

The real-time dynamics of the superconductor is governed by the following time-dependent equations of motion (EOMs):

$$
\begin{gathered}
\partial_{t} \partial_{z} \Phi-i A_{t} \partial_{z} \Phi-\frac{1}{2}\left[f \partial_{z}^{2} \Phi+f^{\prime} \partial_{z} \Phi\right. \\
\left.+i \partial_{z} A_{t} \Phi-z \Phi-A_{x}^{2} \Phi\right]=0, \\
\partial_{t} \partial_{z} A_{t}+2 A_{t}|\Phi|^{2}-i f\left(\Phi^{*} \partial_{z} \Phi-\Phi \partial_{z} \Phi^{*}\right) \\
+i\left(\Phi^{*} \partial_{t} \Phi-\Phi \partial_{t} \Phi^{*}\right)=0,
\end{gathered}
$$

PHYSICAL REVIEW D 93, 121901(R) (2016)

$$
\partial_{t} \partial_{z} A_{x}-\frac{1}{2}\left[\partial_{z}\left(f \partial_{z} A_{x}\right)-2 A_{x}|\Phi|^{2}\right]=0,
$$

combined with the ansatz of the nonvanishing fields $\Phi(t, z)=\Psi(t, z) / z, A_{t}(t, z), A_{x}(t, z)$. There is another constraint equation from the time component of the Maxwell equations,

$$
\partial_{z}\left(\partial_{z} A_{t}\right)-i\left(\Phi^{*} \partial_{z} \Phi-\Phi \partial_{z} \Phi^{*}\right)=0 .
$$

An AC electric field along the $x$ direction can be added by imposing the boundary condition at $z=0$ :

$$
A_{x}(t, z=0)=\frac{E \sin (\omega t)}{\omega} .
$$

Then the electric field is $E_{x}(t)=\partial_{t} A_{x}=E \cos (\omega t)$. The initial condition at $t=0$ is the static solution (time independent) with a fixed chemical potential $A_{t}(z=0)=$ $\mu$ (the dimensionless temperature is defined by $T=3 / 4 \pi \mu$ and the critical values are $\mu_{c}=4.07$ and $T_{c}=0.06$ [25]), which can be obtained by the spectral method. The EOMs will be solved by the fourth-order Runge-Kutta method and the constraint equation is used to monitor the error of the solution, following Ref. [17].

Since the state density near the Fermi surface will oscillate (driven by the oscillating current) and the positive and negative currents cause the same effect, the superconducting gap will oscillate with a frequency twice that of the applied electric field [17,32], as seen in Fig. 1(a). In addition, a small $E$ will hardly affect the superconducting
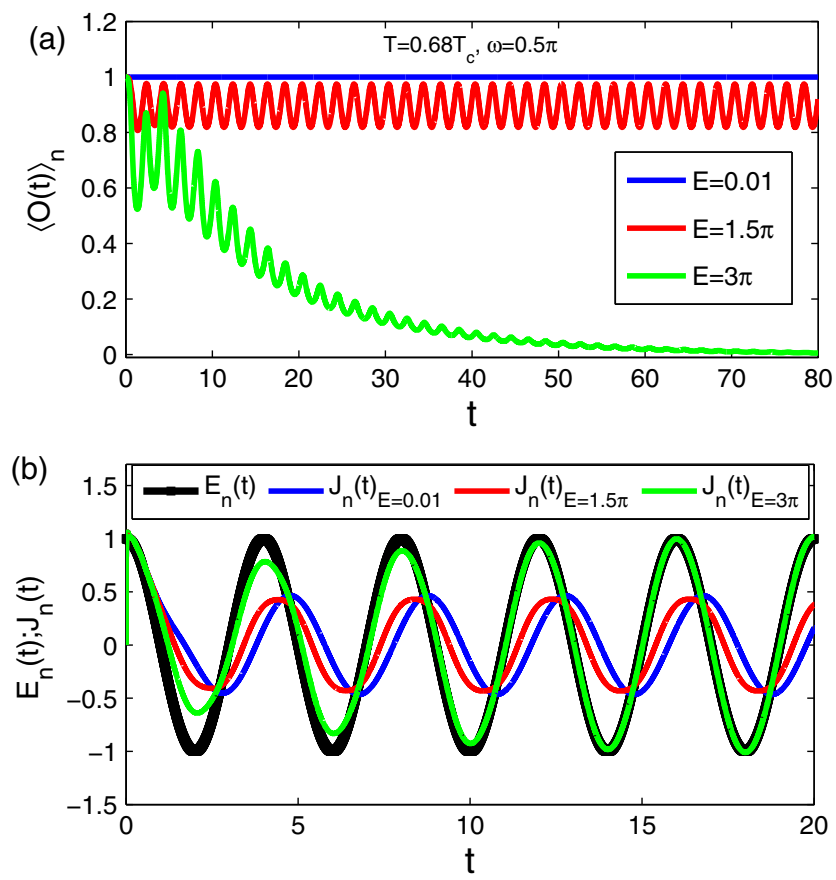

FIG. 1. (a): Superconducting gap dynamics $\langle O(t)\rangle_{n}=\frac{\langle O(t)\rangle}{\langle O(0)\rangle^{\circ}}$ (b): Current dynamics $J_{n}(t)=\frac{J(t)}{J(0)}$. $T=0.68 T_{c}, \omega=0.5 \pi$. 
gap leading the superconductor in a near-equilibrium state. With increasing $E$, the system will be gradually driven out of equilibrium but will still be in a superconducting steady state. Eventually, for a sufficiently large $E$, a nonequilibrium phase transition from a superconducting to a normal state will be induced [17]. At the transition point, the order parameter behaves as $\left(E_{c}-E\right)^{1 / 2}$, where the critical value $E_{c}$ depends on $\mu$ and $\omega$, and at a fixed $\mu, E_{c} \propto \omega$ for large frequency.

The current $J(t)$ is evaluated from Eq. (4), which oscillates with the same frequency as the driving electric field, as shown in Fig. 1(b). The amplitude of the oscillating current $J_{\max }$ reaches a constant value quickly and a timeindependent conductivity is well defined when the current develops a stable form of $J(t)=J_{\max } \sin (\omega t+\theta)$. For the value of $E=3 \pi$, which is above the critical value $E_{c}$, the system enters the nonsuperconducting steady state, and the current is in phase with the electric field (green line), indicating that the conductivity is real. However, for smaller electric fields $E=0.01,1.5 \pi$, where the system is still in a near-equilibrium or out-of-equilibrium superconducting state, the current lags behind the electric field, which indicates that the conductivity is complex.

If we have the real-time dynamics of the current, $\sigma(\omega)$ can be read off from the "ratio" of $J(t)$ to $E(t)$. This enables us to study the regime which is beyond the capability of the linear response theory. From $J(t)=\mathfrak{R}\left[\sigma(\omega) E e^{i \omega t}\right]$ and $E(t)=\Re\left(E e^{i \omega t}\right)=E \cos (\omega t)$, we have

$$
J(t)=E\left[\sigma_{\Re}(\omega) \cos (\omega t)-\sigma_{\Im}(\omega) \sin (\omega t)\right] .
$$

Therefore, the real and imaginary parts of the conductivity can be obtained by fitting the data of $J(t)$ and $E(t)$ after a steady state is achieved.

\section{LINEAR AND NONLINEAR CONDUCTIVITY}

In the weak-field limit $E / \omega \ll\langle O\rangle$, the superconducting gap $\langle O\rangle$ remains a constant in time [blue line in Fig. 1(a)], and the gauge field $A_{x}$ basically can be treated as a perturbation with a negligible backreaction to $A_{t}$ and $\Psi$ [25]. We use Eq. (10) to recompute $\sigma(\omega)$ in the weak-field limit including such a backreaction. The results are shown in Fig. 2, and they agree well with the linear response

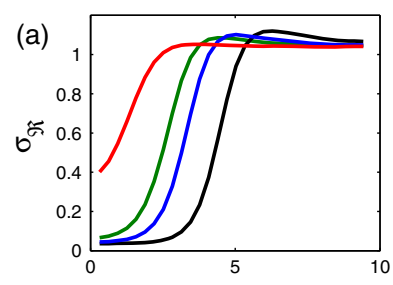

$\omega$

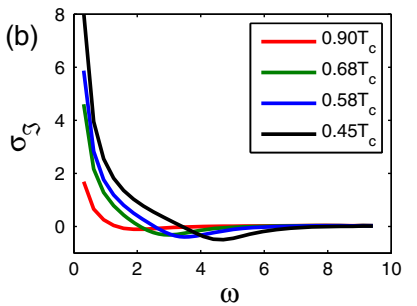

FIG. 2. Real part (a) and imaginary part (b) of the nearequilibrium linear conductivity $\sigma(\omega)$ for different $T$ by fixing $E=0.01 \ll \Delta$. The results are consistent with those in Ref. [25]. results in Ref. [25]. The pole of $\sigma_{\Im}(\omega)$ at $\omega=0$ indicates that the DC conductivity is infinite due to the KramersKronig relation, which is a sign of a superconducting state. The zero DC resistivity can also be observed by studying the $J(t)$ dynamics in a constant electric field by replacing Eq. (9) with $A_{x}(t, z=0)=E t$. We find that in this case $J(t)$ increases linearly in time initially and eventually approaches the critical value at which the superconductivity will be destroyed no matter how small $E$ is. The linear increase of the current in time indicates a zero resistivity according to London's first equation $\partial_{t} J(t) \sim E(t)$. This gives us a hint that there is a critical $\omega$, below which the current will pass its maximum value and then the superconductivity will be destroyed. The minimal frequency we employ here is $\omega=0.1 \pi$, which is larger than the critical value.

The superfluid density $n_{s}$ can be taken as the coefficient of the pole in the imaginary part of the complex conductivity according to

$$
n_{s} \sim \omega \sigma_{\Im}(\omega), \quad \omega \rightarrow 0,
$$

and the normal component is

$$
n_{n} \sim \sigma_{\mathfrak{R}}, \quad \omega \rightarrow 0 .
$$

Moreover, the superconducting gap $\Delta$ can be fitted from the relation $n_{n} \sim e^{-\Delta / T}$, which gives $\Delta=\sqrt{\langle O\rangle} / 2$ [25]. When $\omega \geq 2 \Delta$, the imaginary part falls to zero and the real part approaches one [25]. The normal state without condensation is found to have a constant conductivity $\sigma(\omega)=1$ even for $\omega=0$, which is a well-known result in $\mathrm{AdS}_{4}$ $[25,33]$.

Increasing the electric field to greater than $E=0.01$ will drive the system away from the initial equilibrium state to another steady state [red and green lines in Fig. 1(a)], where the linear response theory is not able to give the correct conductivity. Fortunately, we can still analyze the real-time current dynamics via the holographic duality. It turns out that as the field exceeds a critical value, $E>E_{c}$, the superconductor will finally be driven into the normal conducting state. In this case, the nonequilibrium conductivity is $\sigma(\omega)=1$, which can be clearly seen from the green line in Fig. 1(b) and the large- $E$ regime in Fig. 3. These results agree with previous holographic studies of current dynamics in steady states driven by an external electric field [31], where the dynamical metric driven by a constant electric field is included to accommodate the effect of Joule heating related to the linear growth of the black hole mass.

From Fig. 3(a), the behavior of $\sigma(E)$ with fixed $\omega$ by increasing $E$ can be seen as follows: the real part will finally reach one, while the imaginary part will finally vanish. The $E \rightarrow 0$ results are the same as the equilibrium results in Fig. 2, since we start with an equilibrium superconducting 
ZENG, TIAN, FAN, and CHEN
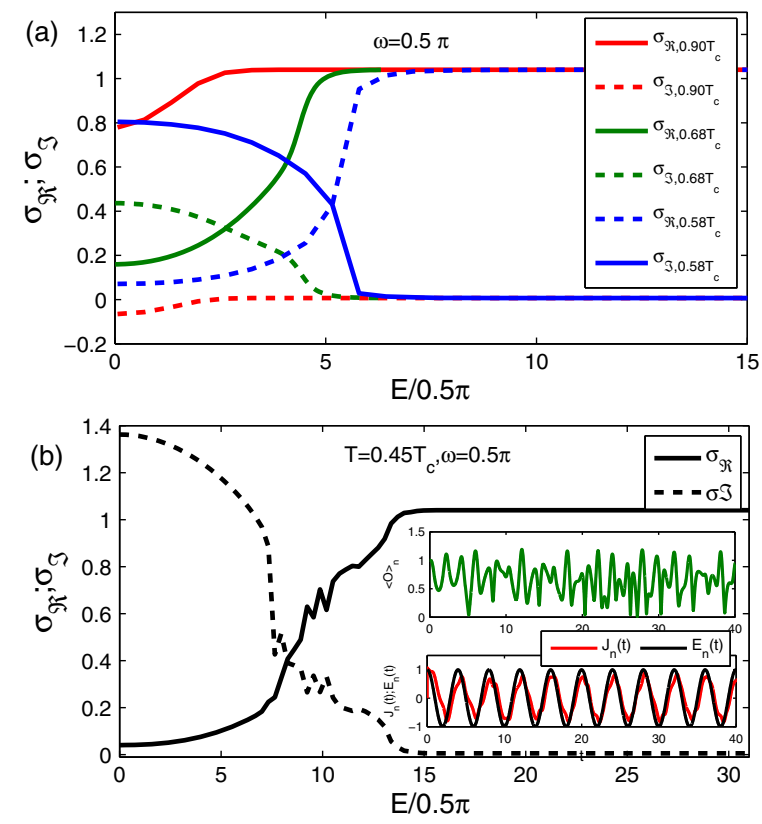

FIG. 3. (a) The behavior of the conductivity $\sigma(E)$ with $\omega=\pi / 2$ at different temperatures. The critical value $E_{c}$ at which the conductivity approaches one increases by decreasing the temperature as a result of a larger superconducting gap. (b) The nonmonotonic dependence of $E$ appears at low temperatures (large $\mu$ ) due to the chaotic response to the electric field.

state when $E$ is small. Actually, three different regimes can be identified: (1) a weak-field regime with linear equilibrium conductivity, which corresponds to a very small range of $E$ near $E=0$; (2) an intermediate-field regime with nonequilibrium nonlinear conductivity, where $E$ is beyond the perturbative limit but smaller than the critical value $E_{c}$; and (3) a large-field regime with nonequilibrium but linear conductivity approaching a constant $\sigma(\omega)=1$.

The nonlinear behavior in the intermediate-field region is rather complicated. The nonlinear conductivity at the lowfrequency limit $\omega \rightarrow 0$ can be explained by a decreased superfluid density $n_{s}$ and an increase of the normal density $n_{n}$. According to Eq. (11) and Eq. (12), the decrease of $n_{s}$ indicates that the imaginary-part pole broadens with increasing $E$ and eventually disappears, while the increase of the normal part $n_{n}$ results in an increase of the real part of the complex conductivity. For large frequency, since the conductivity for small $E$ is already about one, the conductivity basically does not vary as $E$ increases to the nonlinear region. Thus, the significant nonlinear effect is more transparent for nonextremal frequency, and we choose $\omega=$ $0.5 \pi$ for our analysis. Remarkably, at lower temperature $\left(T=0.45 T_{c}\right)$ we have observed a nonsmooth regime where both the superconducting gap and the induced current have chaotic dynamics; see Fig. 3(b). Moreover, in the low- $E$ regime, both the real and imaginary parts of the conductivity with fixed $\omega$ for different temperatures, either close to or far from $T_{c}$, can be fitted by a quadratic polynomial
PHYSICAL REVIEW D 93, 121901(R) (2016)
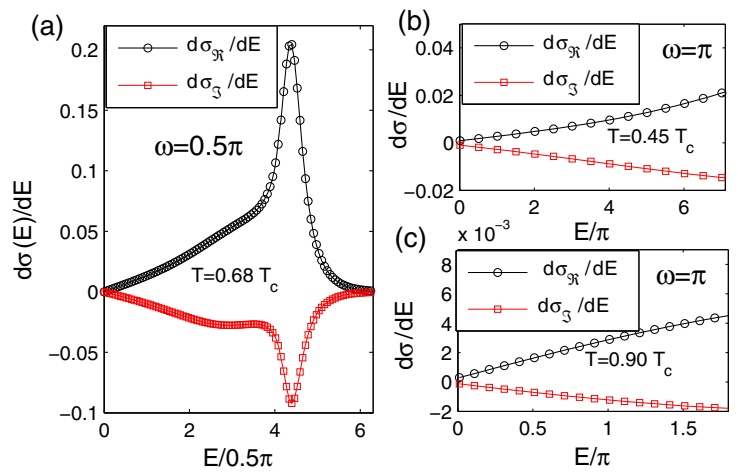

FIG. 4. Linear scaling of $\frac{d \sigma}{d E}=2 a E$ in the low-field regime for different frequencies $\omega$ and temperatures, which confirms the universal $E^{2}$ scaling of the nonlinear conductivity when $E$ is small.

$\sigma(E)=a E^{2}+b$ with two fitting parameters $a$ and $b$ (straight line part of the $d \sigma / d E$ curves in Fig. 4). The parameter $b$ is the value of the linear regime conductivity corresponding to the weak-field limit of $E$. Beyond this part, the system then has very diverse dynamics that significantly depend on the control parameters.

\section{DISCUSSION}

The $E$-dependent conductivity in the intermediate-field superconducting regime clearly cannot be obtained within the linear response theory. Interestingly, a similar $E^{2}$ scaling of the conductivity has been found by Dalidovich and Philips in Ref. [1], where a twodimensional superconductor/insulator phase transition model with the same dynamic critical exponent $z=2$ as the holographic superconductor [34,35] was studied. However, they focused on the DC conductivity on the insulating side, but not on the superconducting side. In fact, the appearance of the nonlinear AC conductivity we have found here can be explained as the suppression of the superconductivity due to the applied electric field $E \cos (\omega t)$. The normal part $n_{n}$ increases while the superconducting part $n_{s}$ decreases with the same scaling of $E^{2}$ with respect to the electric field.

In order to check if these are universal results in any dimensions we should extend the discussion to a $d+1$-dimensional holographic superconductor dual to an $\mathrm{AdS}_{d+2}$ gravity theory. One thing for sure is that in $\mathrm{AdS}_{3}$ and $\mathrm{AdS}_{5}$ the nonlinear conductivity in the nonsuperconducting steady state from a large $E$ is not a constant any more [31].

\section{ACKNOWLEDGMENTS}

We thank Hai Qing Zhang for sharing his code and experiences. We thank Sean A. Hartnoll, Christopher P. Herzog, Shang-Yu Wu and Baruch Rosenstein for valuable comments. H.B.Z. and Z.Y.F. are supported by the 
NONLINEAR TRANSPORT IN A TWO DIMENSIONAL ...

National Natural Science Foundation of China (under Grant No. 11205020 and No. 11404033). Y. T. is partially supported by NSFC with Grant No. 11475179 and the Opening Project of Shanghai Key Laboratory of High
PHYSICAL REVIEW D 93, 121901(R) (2016)

Temperature Superconductors (under Grant No. 14DZ22 60700). C. M. C. is supported by the Ministry of Science and Technology of Taiwan under the grant MOST 1022112-M-008-015-MY3.
[1] D. Dalidovich and P. Phillips, Phys. Rev. Lett. 93, 027004 (2004).

[2] A. G. Green and S. L. Sondhi, Phys. Rev. Lett. 95, 267001 (2005).

[3] A. G. Green, J. E. Moore, S. L. Sondhi, and A. Vishwanath, Phys. Rev. Lett. 97, 227003 (2006).

[4] P. M. Hogan and A. G. Green, Phys. Rev. B 78, 195104 (2008).

[5] A. Karch and S. Sondhi, J. High Energy Phys. 01 (2011) 149.

[6] J. Sonner and A. G. Green, Phys. Rev. Lett. 109, 091601 (2012).

[7] A. M. Berridge and A. G. Green, Phys. Rev. B 88, 220512 (R) (2013).

[8] P. Ribeiro, Q. Si, and S. Kirchner, Europhys. Lett. 102, 50001 (2013).

[9] J. M. Maldacena, Adv. Theor. Math. Phys. 2, 231 (1998).

[10] S. S. Gubser, I. R. Klebanov, and A. M. Polyakov, Phys. Lett. B 428, 105 (1998).

[11] E. Witten, Adv. Theor. Math. Phys. 2, 253 (1998).

[12] O. Aharony, S. S. Gubser, J. M. Maldacena, H. Ooguri, and Y. Oz, Phys. Rep. 323, 183 (2000).

[13] K. Murata, S. Kinoshita, and N. Tanahashi, J. High Energy Phys. 07 (2010) 050.

[14] M. J. Bhaseen, J. P. Gauntlett, B. D. Simons, J. Sonner, and T. Wiseman, Phys. Rev. Lett. 110, 015301 (2013).

[15] X. Gao, A. M. García-García, H. B. Zeng, and H. Q. Zhang, J. High Energy Phys. 06 (2014) 019.

[16] X. Bai, B. H. Lee, L. Li, J. R. Sun, and H. Q. Zhang, J. High Energy Phys. 04 (2015) 066.

[17] W. J. Li, Y. Tian, and H. b. Zhang, J. High Energy Phys. 07 (2013) 030.
[18] P. Basu and S. R. Das, J. High Energy Phys. 01 (2012) 103.

[19] P. Basu, D. Das, S. R. Das, and T. Nishioka, J. High Energy Phys. 03 (2013) 146.

[20] J. Sonner, A. del Campo, and W. H. Zurek, Nat. Commun. 6 , 7406 (2015).

[21] A. M. García-García, H. B. Zeng, and H. Q. Zhang, J. High Energy Phys. 07 (2014) 096.

[22] A. Adams, P. M. Chesler, and H. Liu, Science 341, 368 (2013).

[23] Y. Du, C. Niu, Y. Tian, and H. Zhang, J. High Energy Phys. 12 (2015) 018.

[24] S. S. Gubser, Phys. Rev. D 78, 065034 (2008).

[25] S. A. Hartnoll, C. P. Herzog, and G. T. Horowitz, Phys. Rev. Lett. 101, 031601 (2008).

[26] R. G. Cai, L. Li, L. F. Li, and R. Q. Yang, Sci. China Phys. Mech. Astron. 58, 1 (2015).

[27] S. A. Hartnoll, Classical Quantum Gravity 26, 224002 (2009).

[28] J. McGreevy, Adv. High Energy Phys. 2010, 723105 (2010).

[29] S. Sachdev, Lect. Notes Phys. 828, 273 (2011).

[30] A. G. Green, Contemp. Phys. 54, 33 (2013).

[31] G. T. Horowitz, N. Iqbal, and J. E. Santos, Phys. Rev. D 88, 126002 (2013).

[32] A. Gurevich, Phys. Rev. Lett. 113, 087001 (2014); A. Mitra, S. Takei, Y. B. Kim, and A. J. Millis, Phys. Rev. Lett. 97, 236808 (2006).

[33] C. P. Herzog, P. Kovtun, S. Sachdev, and D. T. Son, Phys. Rev. D 75, 085020 (2007).

[34] K. Maeda, M. Natsuume, and T. Okamura, Phys. Rev. D 79, 126004 (2009).

[35] K. Jensen, Phys. Rev. Lett. 107, 231601 (2011). 